\title{
Measuring rainshafts: Bringing Python to bear on remote sensing data
}

\author{
Scott Collis ${ }^{\S *}$, Scott Giangrande**, Jonathan Helmus ${ }^{\S}$, Di Wull, Ann Fridlind ${ }^{\mathbb{I l}}$, Marcus van Lier-Walquill, Adam \\ Theisen
}

http://www. youtube.com/watch?v=1D0aTToHrCY

\begin{abstract}
Remote sensing data is complicated, very complicated! It is not only geometrically tricky but also, unlike in-situ methods, indirect as the sensor measures the interaction of the scattering media (eg raindrops) with the probing radiation, not the geophysics. However the problem is made tractable by the large number of algorithms available in the Scientific Python community. While SciPy provides many helpful algorithms for signal processing in this domain, a full software stack from highly specialized file formats from specific sensors to interpretable geospatial analysis requires a common data model for active remote sensing data that can act as a middle layer This paper motivates this work by asking: How big is a rainshaft? What is the natural morphology of rainfall patterns and how well is this represented in fine scale atmospheric models. Rather than being specific to the domain of meteorology, we will break down how we approach this problem in terms of the tools used from numerous Python packages to read, correct, map and reduce the data into a form better able to answer our science questions. This is a "how" paper, covering the Python-ARM Radar Toolkit (Py-ART) containing signal processing using linear programming methods and mapping using k-d trees. We also cover image analysis using SciPy's ndimage sub-module and graphics using matplotlib.
\end{abstract}

Index Terms-Remote sensing, radar, meteorology, hydrology

\section{Introduction}

RADARs (RAdio Detection And Ranging, henceforth radars) specialized to weather applications do not measure the atmosphere, rather, the instrument measures the interaction of the probing radiation with the scattering medium (nominally cloud or precipitation droplets or ice particulate matter). Therefore, in order to extract geophysical insight, such as the relationship between large scale environmental forcing and heterogeneity of surface precipitation patterns, a complex application chain of algorithms needs to be set up.

This paper briefly outlines a framework, using a common data model approach, for assembling such processing chains: the Python-ARM Radar Toolkit, Py-ART [Heistermann2014]. This

* Corresponding author: scollis@anl.gov

\& Environmental Sciences Division, Argonne National Laboratory.

**Atmospheric Sciences, Brookhaven National Laboratory.

II NASA Goddard Space Flight Center.

II NASA Goddard Institute of Space Sciences.

+University of Oklahoma, Cooperative Institute for Mesoscale Meteorological Studies, ARM Climate Research Facility Data Quality Office.

Copyright () 2014 Scott Collis et al. This is an open-access article distributed under the terms of the Creative Commons Attribution License, which permits unrestricted use, distribution, and reproduction in any medium, provided the original author and source are credited. paper also provides an example application: using rainfall maps to objectively evaluate the skill of fine scale models in representing precipitation morphology.

\section{The data source: scanning centimeter wavelength radar}

Rainfall can occur at many different scales. From small, descrete storm cells at scales of 10's of kilometers to to large scale tropical systems such as hurricanes which cover 100's to 1000's of kilometers. Some complex systems can contain many scales and in order to understand this spatial complexity of precipitating cloud systems a sensor is required that can collect spatially diverse data. Radars emit a spatially discrete pulse of radiation with a particular beamwidth and pulse length. A gated receiver detects the backscattered signal and calculates a number of measurements based on the radar spectrum (the power as a function of phase delay which is due to the motion of the scattering medium relative to the antenna). These moments include radar reflectivity factor $Z_{e}$, radial velocity of the scattering medium $v_{r}$ and spectrum width $w$. Polarimetric radars transmit pulses with the electric field vector horizontal to the earth's surface as well as vertical to the earth's surface. These radars can give a measure of the anisotropy of the scattering medium with measurements including differential reflectivity $Z_{D R}$, differential phase difference $\phi_{d p}$ and correlation coefficient $\rho_{H V}$. The data is laid out on a time/range grid with each ray (time step) having an associated azimuth and elevation. Data presented in this paper are from 4 ARM [Mather2013] radar systems: One C-Band (5 cm wavelength) and three X-Band $(3 \mathrm{~cm}$ wavelength) radars as outlined in table 1.

These instruments are arranged as show in figure 1.

\section{The Python ARM Radar Toolkit: Py-ART}

Radar data comes in a variety of binary formats but the content is essentially the same: A time-range array for each radar moment along with data describing the pointing and geolocating of the platform. For mobile radar the platform's motion must also be described in the file. Py-ART takes a common data model approach, carefully designing the data containers and mandating that functions and methods accept the container as an argument and return the same data structure. The common data model for radar data in Py-ART is the Radar class which stores data and metadata in Python dictionaries in a particular instance's attributes. Data is 


\begin{tabular}{lll}
\hline & X-SAPR & C-SAPR \\
Frequency & $9.4 \mathrm{GHZ}$ & $6.25 \mathrm{GHz}$ \\
Transmitter & Magnetron & Magnetron \\
Power & $200 \mathrm{~kW}$ & $350 \mathrm{~kW}$ \\
Gate spacing & $50 \mathrm{~m}$ & $120 \mathrm{~m}$ \\
Maximum & $40 \mathrm{~km}$ & $120 \mathrm{~km}$ \\
Range & & $1^{\circ}$ \\
Beam width & $1^{\circ}$ & Simul. H/V \\
Polar. mode & Simul. H/V & Adv. Radar Corp. \\
Manufacturer & Radtec & NCAR MDV \\
Native format & Iris Sigmet & \\
\hline
\end{tabular}

TABLE 1: ARM radar systems used in this paper.

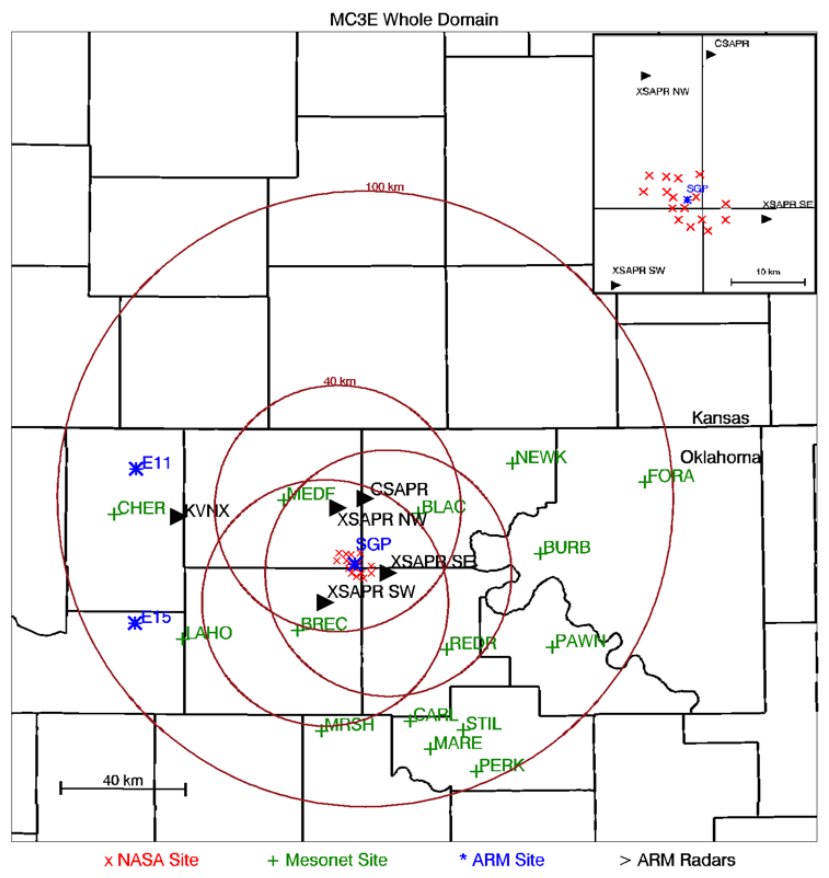

Fig. 1: Arrangement of radars around the ARM Southern Great Plains Facility from [Giangrande2014].

stored in a NumPy array in the 'data' key of the dictionary. For example:

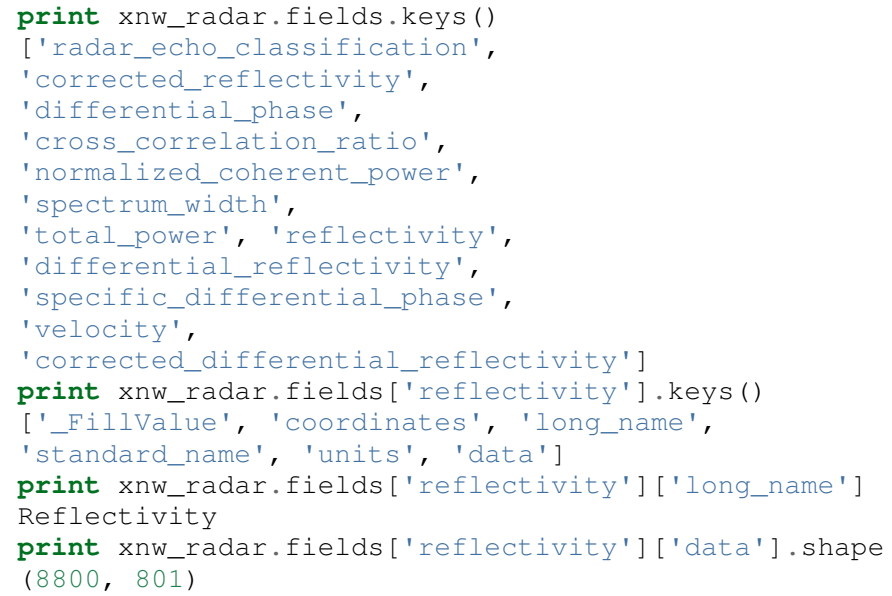

The xnw_radar has a variety of fields, including 'reflectivity' with the numerical moment data stored in the 'data' key with 8800 time steps and 801 range gates. Data on instrument pointing is stored in

\begin{tabular}{lll}
\hline $\begin{array}{l}\text { Format name } \\
\text { CF-Radial }\end{array}$ & $\begin{array}{l}\text { Example radar system(s) } \\
\text { NCAR SPOL, ARM Cloud }\end{array}$ & $\begin{array}{l}\text { Note } \\
\text { Output format }\end{array}$ \\
RF & $\begin{array}{l}\text { Lots of legacy data } \\
\text { BoM CPOL in Darwin, Aus- }\end{array}$ & Via RSL \\
Lassen & $\begin{array}{l}\text { Via RSL } \\
\text { tralia }\end{array}$ & Native \\
IRIS Sigmet & ARM X-SAPR & Native \\
NCAR MDV & ARM C-SAPR & Native \\
GAMIC & European radar network & Native \\
WSR-88D & USA operational network & Native \\
CHILL & NSF funded deployable S- & \\
& Band & \\
\hline
\end{tabular}

TABLE 2: Py-ART formats.

x_nw.azimuth and $\mathrm{x} \_n w . e l e v a t i o n$ attributes while the center point of each range gate is stored in $\mathrm{x} \_n w$. range. Again these attributes are dictionaries with data stored in the 'data' key. Functions in Py-ART can append fields or modify data in existing fields (rare).

The vital key is a 'Babelfish' layer which ingests a variety of formats into the common data model. Currently table 2 outlines the formats which are compatible with Py-ART. A number of these formats are available via a Cython wrapper around NASA's Radar Software Library.

There is also active development on supporting NOAA NOX$P$ and NASA D3R radars. Py-ART supports a single output format for radial geometry radar data which is, CF-Radial. CF-Radial is a NetCDF based community format on which the common data model in Py-ART is based on.

Py-ART forms part of an ecosystem of open source radar applications, many of which are outlined in [Heistermann2014]. A key challenge for the radar community is reaching consensus on data transport layers so that an application chain can be built using multiple applications. In terms of the rest of the Scientific python ecosystem, Py-ART brings the data into Python in a very simple way so users can simply and quickly get to doing Science.

\section{Pre-mapping corrections and calculations}

Once raw data is collected there is often a number of processing steps that need to be performed. In our case this includes:

- Correcting false Azimuth readings in the Northwest XBand system.

- Cleaning data of undesirable components such as multiple trips, clutter and non-meteorological returns.

- Processing the raw $\phi_{D P}$ and extracting the component due to rain water content by using a linear programming technique to fit a profile which mandates positive gradient, see [Giangrande2013].

- Using reflectivity and $\phi_{D P}$ to retrieve attenuation (in $\mathrm{dBZ} / \mathrm{km}$ ) due to rainwater path.

- Using the techniques outlined in [Ryzhkov2014] to retrieve rainfall rate (in $\mathrm{mm} / \mathrm{hr}$ ) from attenuation.

These are all outlined in the first of the three notebooks which accompany this manuscript: http://nbviewer.ipython.org/ github/scollis/notebooks/tree/master/scipy2014/. Each process either appends a new field to the Radar instance or returns a field dictionary which can then be added to the instance. Py-ART also comes with visualization methods allowing for the conical (or Plan Position Indicator, PPI) scan to be plotted and geolocated 

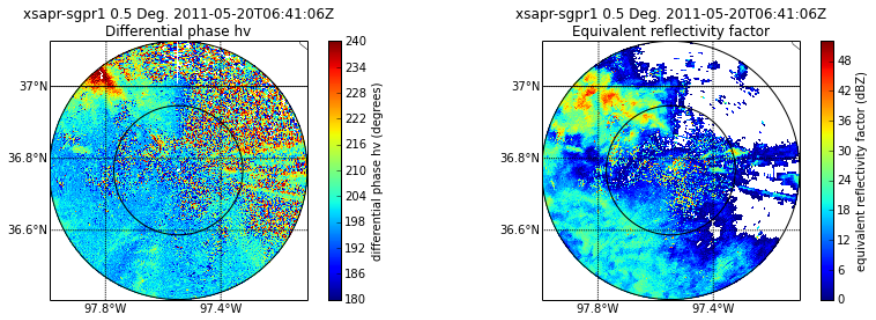

Fig. 2: Raw reflectivity factor and polarimetric phase difference from the lowest (0.5 degree) tilt.

using matplotlib and Basemap. An example plot of raw $\phi_{D P}$ and reflectivity is shown in figure 2 .

The code necessary to create this plot:

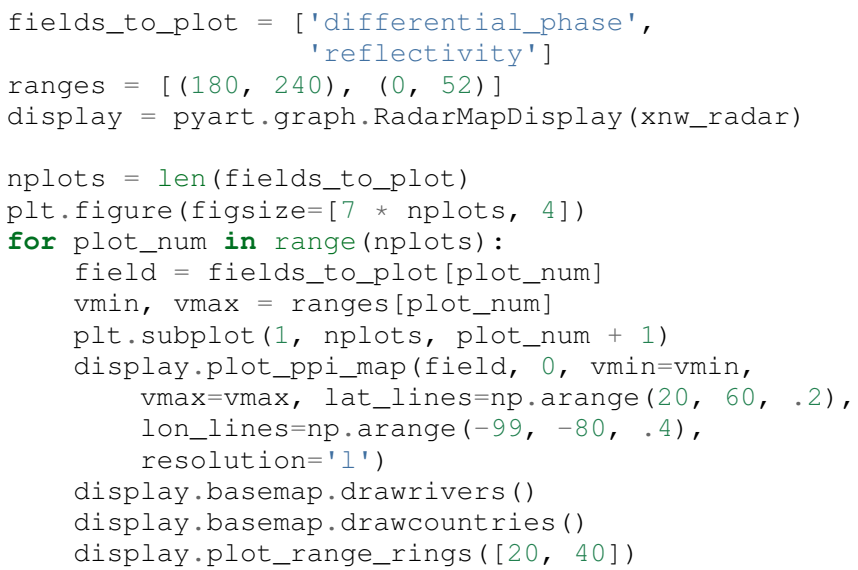

Here, a RadarMapDisplay instance is instantiated by providing a Radar object which is insensitive to the data source. The sample plotting routines can be used to plot data ingested from any of the formats which Py-ART supports.

\section{Mapping to a Cartesian grid}

Radars sample in radial coordinates of elevation, azimuth and range. Mathematics for atmospheric phenomena are greatly simplified on Cartesian and Cartesian-like (eg pressure surfaces) grids. Therefore the raw and processed data in the Radar object often need to be mapped onto a regular grid. In the field, this is known as "Objective analysis" (see, for example [Trapp2000]). In this paper we use a technique known as Barnes analysis [Barnes1964] which is an inverse distance weighting, sphere of influence based technique. For each grid point in the Cartesian grid a set of radar gates within a radius of influence are interpolated using the weighting function:

$$
W(r)=e^{\frac{-r_{i n f l}^{2}}{2.0 * r^{2}}}
$$

where $r$ is the distance from the grid point and $r_{\text {infl }}$ is the search radius of influence. A brute force method for performing this mapping would be to calculate the distance from each Cartesian point to each radar gate to find those within the radius of influence, a method which scales as $n * m$ where $n$ is the number of points in the grid and $m$ the number of gates in the radar volume. With a typical grid being 200 by 200 by 37 points and a modern radar having on the order of 8000 time samples and 800 range gates this quickly becomes intractable. A better method is to store the radar gates in a k-d tree or related data structure. This reduces the search to an order $n * \log (m)$ problem. This method is implemented in Py-ART.
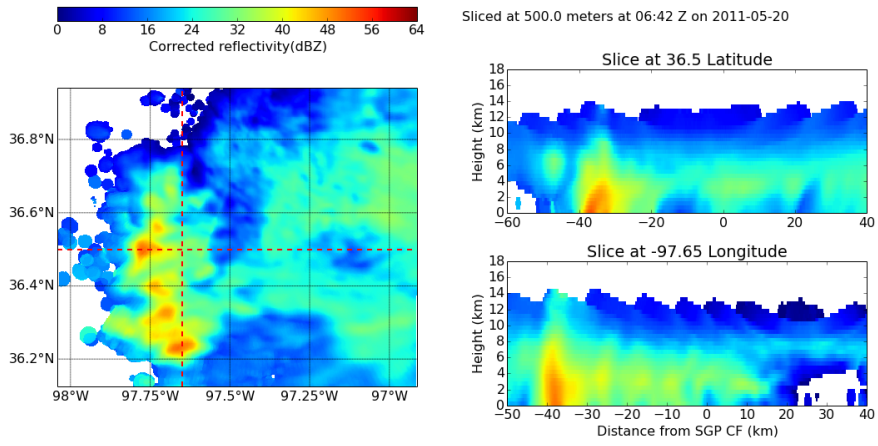

Fig. 3: Single C-Band reflectivity factor field.

In addition a variable radius of influence algorithm is implemented which analyzes the radar volume coverage pattern and deduces an optimized $r_{\text {infl }}$ at each grid point. Unlike many other objective analysis codes Py-ART implementation can operate on multiple Radar objects simultaneously, treating the radar gates as a cloud of points. This allows the merging of multiple radar data sets. The method is simple to invoke, for example the code snippet:

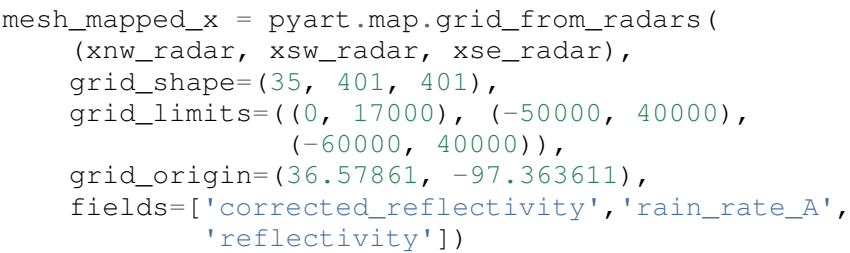

will map the gates in the three Radar objects (in this case the three ARM X-Band systems in figure 1$)$ to a grid that is $(\mathrm{z}, \mathrm{y}, \mathrm{x})=(35$, 401,401 ) points with a domain of 0 to $17 \mathrm{~km}$ in altitude, -50 to $40 \mathrm{~km}$ in meridional extend and -60 to $40 \mathrm{~km}$ in zonal extent. The method returns a Grid object which follows a similar layout to a Radar object: fields are stored in the fields attribute, geolocation data in the axes attribute with the numerical data found in the 'data' key of the dictionaries.

Again, as with the Radar object Py-ART has a menu of available routines to visualize data contained in Grid objects as well as an input output layer that can inject CF-compliant netCDF grids and write Grid object out to a CF-complaint file for future analysis and distribution.

For example figure 3 shows a slice through mapped reflectivity from the ARM C-SAPR at $500 \mathrm{~m}$ and cross sections at $36.5 \mathrm{~N}$ degrees latitude and $-97.65 \mathrm{E}$ longitude.

In the vertical cross sections clear artifacts can be seen due to the poor sampling. Figure 4 shows the same scene but using a grid created from three X-Band radars in a network. In both figures the radar data are mapped onto a grid with $225 \mathrm{~m}$ spacing.

It is clear that more fine scale detail is resolved due to the rain systems being closer to any given radar in the X-Band network grid. In addition, due to the higher density of high elevation beams (essentially a "web" of radar beams sampling the convective anvil) sampling artifacts are greatly reduced and finer details aloft are able to be studied.

Mesh mapping only works for "specific" measurements, ie not integrated measurements like $\phi_{D P}$ or directionally dependent moments like $v_{r}$. One measurement that can be mapped is our retrieved rain rate.

Figures 5 and 6 show mappings for rain rate using just the CBand measurement and X-Band network respectively. Again the 


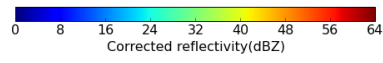

Sliced at 500.0 meters at 06:41 Z on 2011-05-20
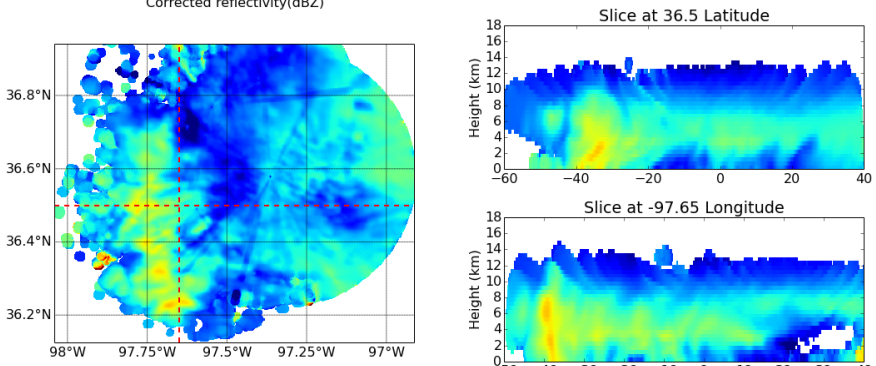

Slice at -97.65 Longitude

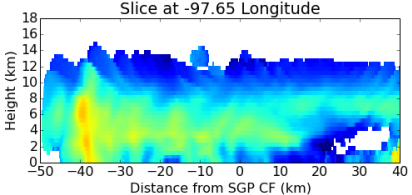

Fig. 4: Reflectivity factor mapped from a network of X-Band radars.
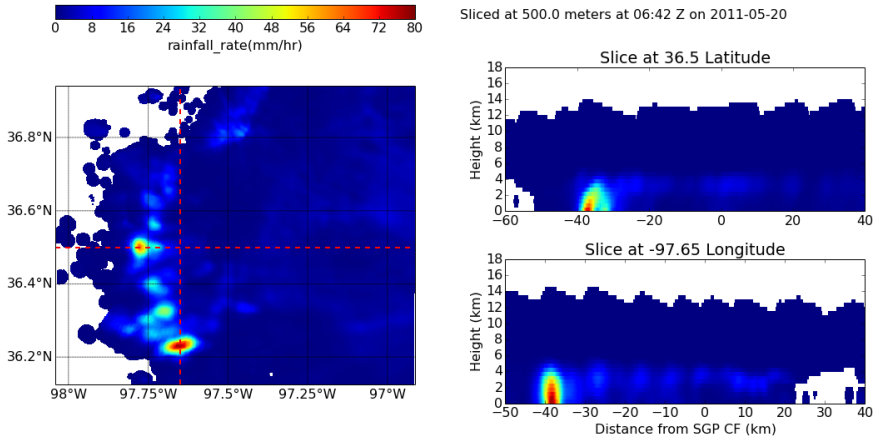

Fig. 5: Single C-Band rainfall field.

mesh map of the X-Band retrieval shows very fine detail resolving (in a volumetric dataset) fall streak patterns. The maxima near $4 \mathrm{~km}$ (just below the freezing level) is due to melting particles. The rainfall retrieval has a cut off at the sounding determined freezing level but the "bright band" can extend some depth below this. Future work will entail using polarimetric measurements to determine where there is only pure liquid returns and conditionally apply the rainfall retrieval to those positions.

\section{Spatial distribution of rainfall: a objective test of fine scale models}

Previous sections have detailed the correction, retrieval from and mapping to a Cartesian grid of radar data. The last section showed enhanced detail can be retrieved by using a network of radars. The question remains: how can the detail in rain fields be objectively compared? Can parameters derived from radar data be compared to those calculated from forecast models? The meshes generated
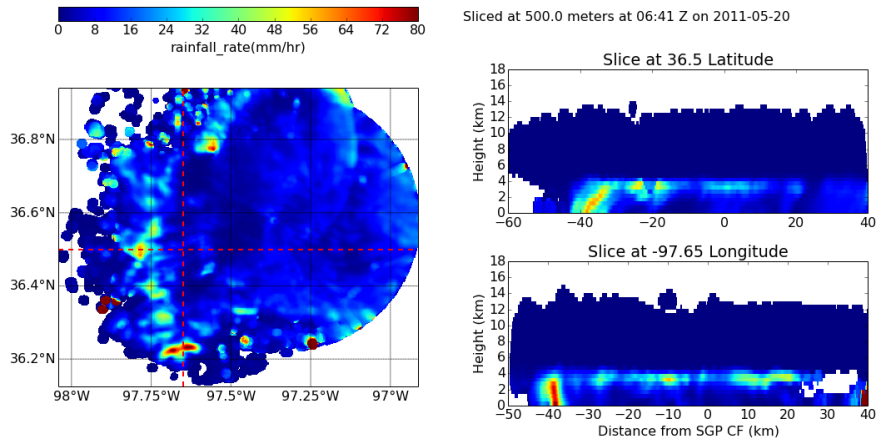

Fig. 6: Rainfall from a network of X-Band systems.

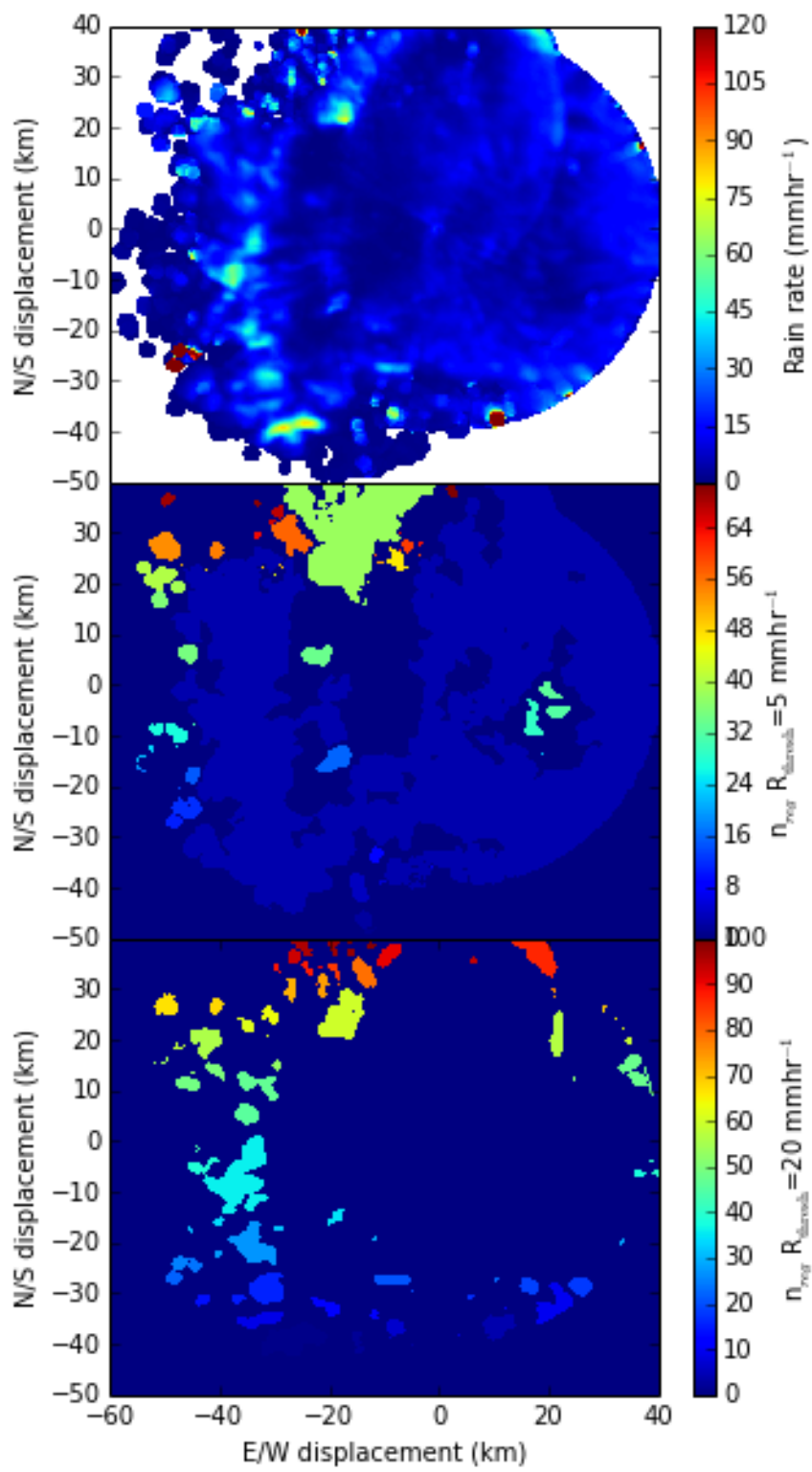

Fig. 7: An example of figure segmentation using scipy.ndimage.label.

using the mapping techniques previously discussed can be treated like image data for which a number of packages exist for analysis.

\section{Measuring rainshafts using SciPy's ndimage subpackage}

A simple technique for documenting the features present in an image is to partition it into segments which are above a certain threshold and calculate the number of segments, their accumulated area and the mean rainfall across the segment. The ndimage subpackage in Scipy is perfect for accomplishing this. Figure 7 shows the use of scipy.ndimage.label to segment regions above 5 and $20 \mathrm{~mm} / \mathrm{h}$.

The code is very simple, for a given rain rate it creates a "black and white" image with whites above the threshold point and the black below, then scipy.ndimage.label segments the regions into a list of regions from which metrics can be calculated:

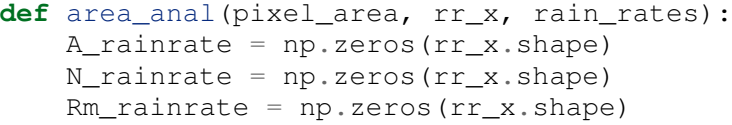




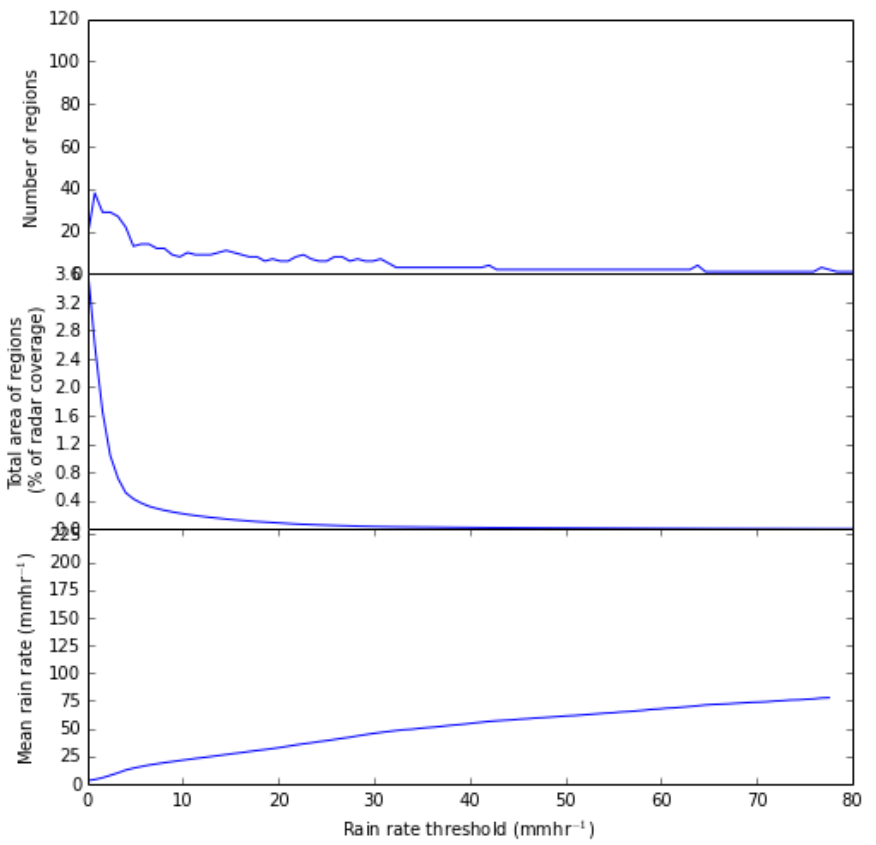

Fig. 8: Number of regions, region covered and mean rain rate as a function of rain rate threshold for a rainmap produced by a single C-Band system.

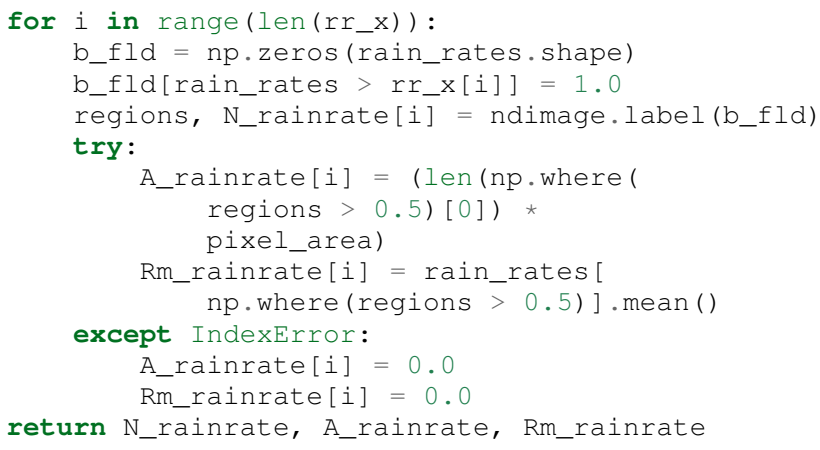

This produces plots for the $\mathrm{X}$-Band mesh as seen in 9 and single C-Band sytems in 8.

The results presented in this paper show that the rainfall field for this case is under-resolved when observed by a single C-Band system. While we have not established that a nework of X-Band systems fully resolve the spatial complexity of the rainfall field it clearly shows more detail, especially at higher altitudes.

Future work will focus on establishing limits to spatial complexity and understanding how large scale forcing (instability, mouisture etc) influence complexity. In addition we will be applying this technique to fine scale model data as an "observational target" for the model to achieve. That is the methodes outlined in this paper can be used as a simple optimization metric which can be used when adjusting the parameters in a model.

\section{Conclusions}

This paper has covered the pipeline for proceeding from raw radar measurements through quality control and geophysical retrieval to mapping and finally to the extraction of geophysical insight. The simple conclusion is that, with careful processing, a network of X-Band radars can resolve finer details than a single C-Band radar. More importantly, finer details exist. The paper also presents

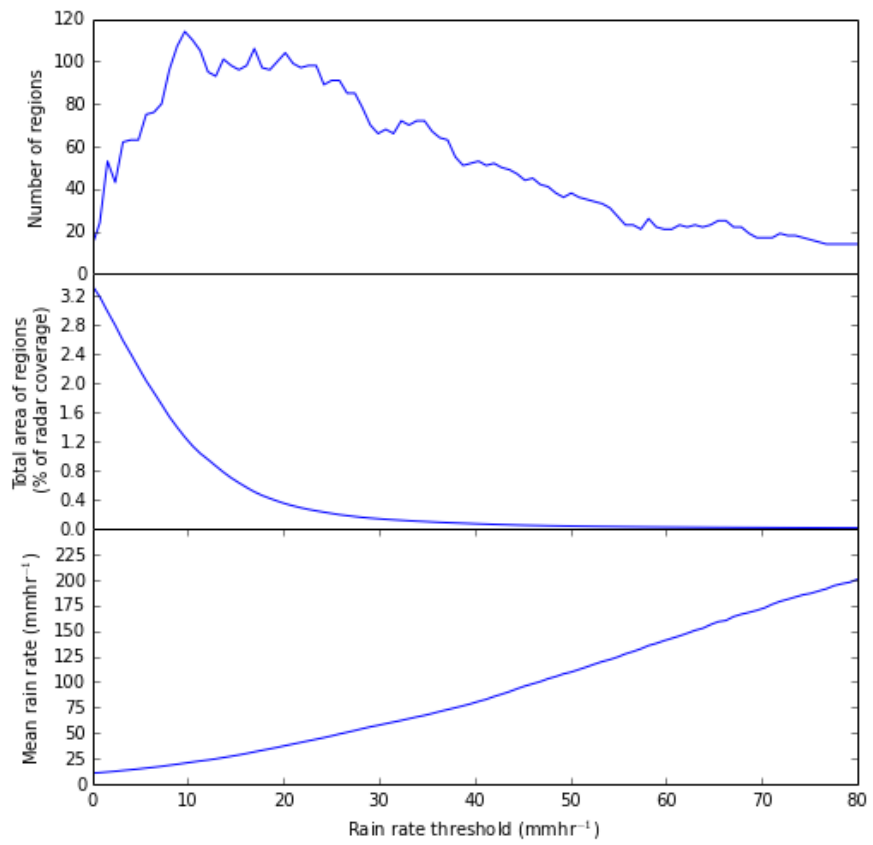

Fig. 9: Number of regions, region covered and mean rain rate as a function of rain rate threshold for a rainmap produced by a network of $X$-Band systems.

a very simple, image processing based technique to take the "morphological finger print" of rainfall maps. This technique can be used on both remotely sensed and numerically modeled data providing a objective basis for model assessment.

\section{Acknowledgements}

Dr. Giangrande's work is supported by the Climate Science for a Sustainable Energy Future (CSSEF) project of the Earth System Modeling (ESM) program in the DOE Office of Science. Argonne National Laboratory's work was supported by the U.S. Department of Energy, Office of Science, Office of Biological and Environmental Research (OBER), under Contract DE-AC0206CH11357.The work has also been supported by the OBER of the DOE as part of the ARM Program. Adam Theisen's work was supported by Battelle - Pacific Northwest National Laboratory, contract number 206248, and his home institution, CIMMS, is supported by NOAA/Office of Oceanic and Atmospheric Research under NOAA-University of Oklahoma Cooperative Agreement \#NA11OAR4320072, U.S. Department of Commerce. The authors wish to thank Dr. Alexander Ryzhkov for support on implementation of specific attenuation-based rainfall methods. We would also like to thank the reviewers of this paper, James Bergstra and Terry Letsche.

\section{REFERENCES}

[Heistermann2014] Heistermann, M., S. Collis, M. J. Dixon, S. E. Giangrande, J. J. Helmus, B. Kelley, J. Koistinen, D. B. Michelson, M. Peura, T. Pfaff and D. B. Wolff, 2014: The Promise of Open Source Software for the Weather Radar Community. Bull. Amer. Meteor. Soc., In Press.

[Mather2013] Mather, J. H., and J. W. Voyles, 2012: The Arm Climate Research Facility: A Review of Structure and Capabilities. Bull. Amer. Meteor. Soc., 94, 377-392, doi:10.1175/BAMS-D-11-00218.1. 
[Giangrande2014] Giangrande, S. E., S. Collis, A. K. Theisen, and A. Tokay, 2014: Precipitation Estimation from the ARM Distributed Radar Network During the MC3E Campaign. J. Appl. Meteor. Climatol., doi:10.1175/JAMC-D-13-0321.1. http://journals.ametsoc.org/doi/abs/10.1175/JAMC-D-130321.1

[Giangrande2013] Giangrande, S. E., R. McGraw, and L. Lei, 2013: An Application of Linear Programming to Polarimetric Radar Differential Phase Processing. Journal of Atmospheric and Oceanic Technology, 30, 1716-1729, doi:10.1175/JTECH-D-12-00147.1.

[Ryzhkov2014] Ryzhkov, A. V., M. Diederich, P. Zhang, C. Simmer, 2014: Potential utilization of specific attenuation for rainfall estimation, mitigation of partial beam blockage, and radar networking. Submitted, J. Atmos. Oceanic Technol., in press.

[Trapp2000] Trapp, R. J., and C. A. Doswell, 2000: Radar Data Objective Analysis. Journal of Atmospheric and Oceanic Technology, 17, 105-120, doi:10.1175/15200426(2000)017<0105:RDOA>2.0.CO;2.

[Barnes1964] Barnes, S. L., 1964: A Technique for Maximizing Details in Numerical Weather Map Analysis. Journal of Applied Meteorology, 3, 396-409, doi:10.1175/15200450(1964)003<0396:ATFMDI>2.0.CO;2. 